\title{
THE INHIBITORY EFFECT OF TRIAMCINOLONE ON ADRENAL FUNCTION
}

\author{
BY \\ C. B. HATFIELD* AND S. SHUSTER $\dagger$ \\ From the Department of Medicine, Postgraduate Medical School of London
}

(RECEIVED FOR PUBLICATION SEPTEMBER 9, 1958)

Many steroid hormones will depress the secretion of hydrocortisone by the adrenal by inhibiting secretion of pituitary corticotrophin. This inhibition has proved a useful means of demonstrating an intact pituitary-adrenal axis, and may help to distinguish between adrenal cortical neoplasia and hyperplasia (Jailer, Gold, and Wallace, 1954 ; Cope, 1956). However, most of these hormones are of little use for this purpose because suppressive doses contribute significantly to the metabolites whose measurement in urine and plasma is used as an index of adrenal activity. With the introduction of the more potent steroid, 9-alpha-fluoro-hydrocortisone, the dose required to produce adrenal depression was considerably reduced, and the interference with steroid analysis produced by metabolites of the administered hormone was therefore less. The 16-hydroxylated derivative of 9 - alpha - fluoro-hydrocortisone, "triamcinolone" (9 - alpha - fluoro - 16 - alpha hydroxyl-delta-hydrocortisone acetate), is reported (Bernstein, Lenhard, Allen, Heller, Littell, Stolar, Feldman, and Blank, 1956) to have less glucocorticoid activity than its parent hormone. This suggests that larger doses would be required to produce adrenal suppression (DiRaimondo and Forsham, 1958), which in consequence may interfere with measurement. However, plasma hydrocortisone concentrations in two patients receiving the hormone therapeutically were observed to be well below normal levels. This was particularly surprising in view of the chemical structure of the compound, and led to the present investigations in man of the ability of "triamcinolone" to depress adrenal secretion, and an assessment of its suitability for clinical use in adrenal function tests.

\section{Methods}

Plasma hydrocortisone was determined by the method of Peterson, Karrer, and Guerra (1957) with the following modifications.

\footnotetext{
* Present address: Department of Medicine, University of Minnesota Hospitals.

$\uparrow$ Present address: Medical Unit, Cardiff Royal Infirmary.
}

(1) Methylene dichloride was shaken twice with concentrated sulphuric acid, neutralized with sodium hydroxide, washed four to five times with water, and redistilled.

(2) Phenylhydrazine was dissolved in ethanol and treated with activated charcoal. It was recrystallized after filtration.

(3) The extraction technique was simplified by vigorously shaking the plasma and methylene dichloride for one minute in 8-in. "quickfit" stoppered tubes. No trouble was experienced with emulsions.

Procedures (1) and (2) reduced the reagent blank considerably. The plasma was separated immediately after collection and frozen until analysed. Urinary 17, 21 di-hydro-20-ketosteroids were determined before and after hydrolysis with rat $\beta$ glucuronidase by the method of Silber and Porter (1954) as modified by Liddle, Richard, and Peterson (1955).

Urinary 17-ketosteroids were measured as recommended by the M.R.C. (1951) and 17-ketogenic steroids and "total 17-hydroxycorticosteroids" were determined by the methods of Norymberski (Norymberski, Stubbs, and West, 1953 ; Appleby, Gibson, Norymberski, and Stubbs, 1955). All urines were collected without preservative and refrigerated until analysed.

Two subjects, P.T., a girl with cirrhosis without ascites aged 16 years, and C.H., a normal male aged 31 years, were studied in detail. Toxic side-effects of " triamcinolone" permitted only control observations on a third subject, who was therefore excluded from the study. During the observation period, daily 24-hour urine specimens were collected (beginning at 9 a.m.) and blood specimens were obtained at the end of each urine collection interval. After a two-day control period, "triamcinolone" (8 mg., q.d.s.) was administered for 11 and five days respectively. During the last two days of " triamcinolone" administration, 100 i.u. of A.C.T.H. gel (Armour) was given intramuscularly at 9 a.m. Plasma hydrocortisone was measured in blood drawn at 9 a.m. from two cirrhotic patients, who had been receiving "triamcinolone" (8 mg. q.d.s.) for eight days.

\section{Results}

The ability of pure "triamcinolone" to react in the above-noted techniques was studied. The 
Subject P. T.

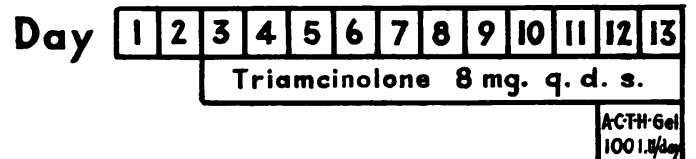

15 Plasma Hydrocortisone Concentration

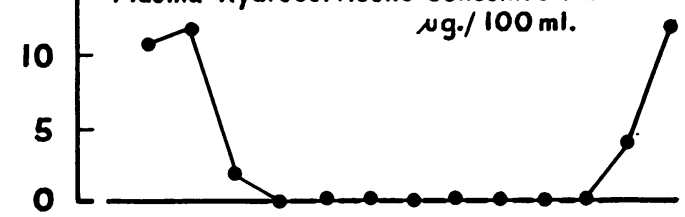

$\neg 5$ Conjugaled Porter-silber Steroids

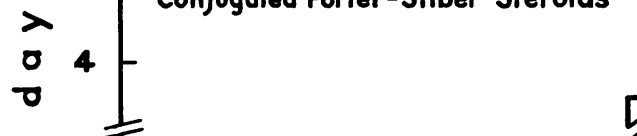

1

a 0

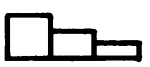

ơ 3.0 c Free Porter-Silber Steroids $\underbrace{\infty}_{1.0} 1.5 \frac{F}{F}$

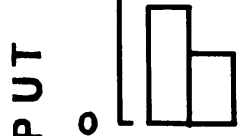

a 0
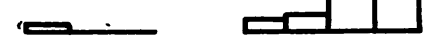

- 10 工 Total 17.0H Corticosteroids

- 4 F

0
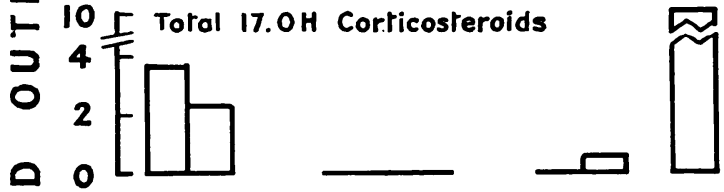

$\overline{0}$

o

2 17 Kerogenic Steroids

U

1

$\boldsymbol{n}$
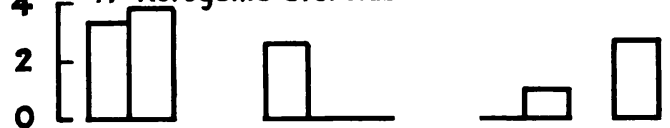

$>$

10 工 17 Kelosteroids

$22 \frac{1}{7}$

$\begin{array}{ll}\approx & 1 \\ 2 & 0\end{array}-\square$

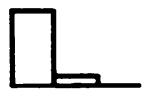

Urine Outpur [litres]

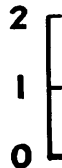

${ }_{0}^{2}[$

Plasma hydrocortisone concentrations measured at 9 a m.

a.m. and 24-hour urinary corticosteroid outputs A.C.T.H. gel.
Subject C.H.
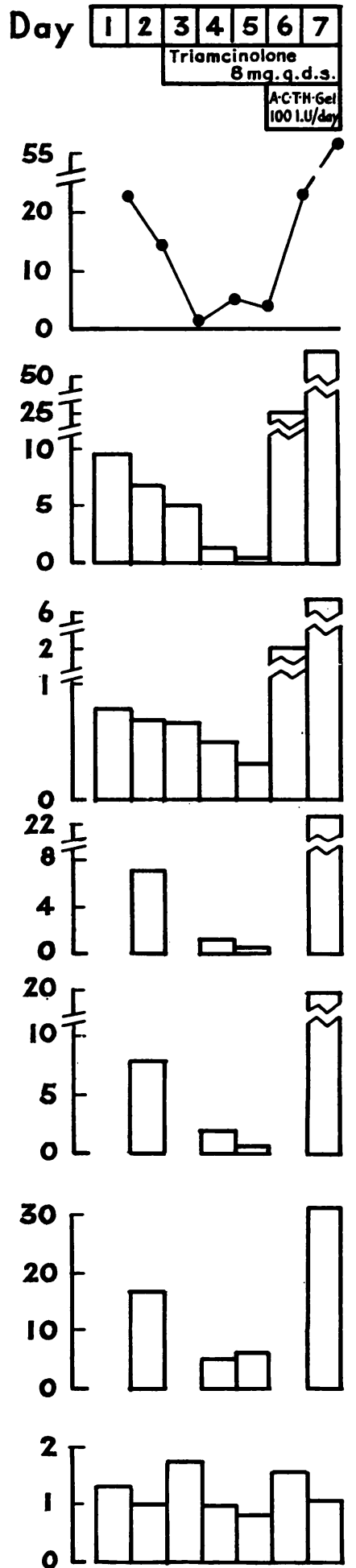
chromogenicity of "triamcinolone" compared on a weight for weight basis was no greater than $1 \%$ of that of dehydroisoandrosterone using the techniques measuring 17-ketosteroids and 17-ketogenic steroids, and $3 \%$ for the "total 17-hydroxycorticosteroids." The direct Zimmerman test gave no detectable reaction. Chromogenicity by the Porter-Silber technique was $3 \%$ of that of hydrocortisone.

After seven days of "triamcinolone" therapy the two cirrhotic patients had plasma hydrocortisone concentrations of 0.6 and $1.6 \gamma \%$ respectively at 9 a.m., which is well below the lower limit of normal, i.e., 6-25 $\mu \mathrm{g}$. \%, for the method (Peterson et al., 1957).

The results of the detailed studies on the two subjects are presented in Fig. 1. The plasma hydrocortisone concentration fell to subnormal levels after the administration of "triamcinolone," and a parallel reduction in urinary steroid output was observed. The data further show that suppression was not maximal until after three days of receiving "triamcinolone." Both plasma hydrocortisone concentration and urinary steroid output increased rapidly after A.C.T.H. therapy. In P.T. the daily urinary output during "triamcinolone" administration was consistently higher than output during the whole period, while in C.H. no consistent change in urinary output was observed. It should be noted that the basal steroid excretion in P.T. was low. This was probably associated with the liver disorder, though her age and sex may have been contributory factors.

Toxic effects, consisting of lightheadedness, nausea, and insomnia, made it necessary to discontinue the use of the drug in one subject not reported here. In the other subjects no untoward effects were noted.

\section{Discussion}

The observation that " triamcinolone" produces little Porter-Silber chromogen is of considerable interest as this hormone possesses a 17, 21, dihydroxy-20-ketosteroid configuration which is characteristic of Porter-Silber reacting compounds (Silber and Porter, 1954). The hydroxyl group on C 16 may account for the relative inactivity of this steroid in this reaction. Because this hormone does not significantly interfere with commonly used measuring techniques, study of its effects on adrenal function is simplified.

The present findings of markedly reduced plasma hydrocortisone and diminished urinary corticosteroid output indicate adrenal depression during "triamcinolone" administration. The prompt rise of plasma hydrocortisone and urinary steroids following A.C.T.H. gel given simultaneously with "triamcinolone" suggests that this hormone does not impair the adrenal responses to A.C.T.H. It appears, therefore, that "triamcinolone" acts similarly to other adrenal corticoids, such as cortisone, by inhibiting pituitary A.C.T.H. production (Sayers and Sayers, 1948). The delay before the maximal adrenal depression by "triamcinolone" indicates that for clinical testing the steroid should be administered for a period of not less than three days.

\section{Summary}

The effect of a new synthetic hormone, "triamcinolone," on adrenal function was investigated.

"Triamcinolone," in the dose employed, did not produce a significant amount of urinary or plasma chromogen with any of the commonly employed techniques for measuring corticosteroids.

Marked depression of adrenal function was found in two subjects studied while they were receiving the drug. The response to A.C.T.H. gel while the subjects were receiving "triamcinolone" indicated that the suppressive effect was not a direct action of the steroid on the adrenals.

It is concluded that "triamcinolone" is suitable for clinical use in tests of adrenal inhibition.

The present work was carried out while in receipt of a MacEachem fellowship from the Canadian Cancer Society (C. B. Hatfield) and a personal grant from the Medical Research Council (S. Shuster).

The authors wish to thank Dr. S. Sherlock for permission to investigate one of her patients, and Dr. C. L. Cope for his guidance and helpful criticism of the text and for laboratory facilities.

We are grateful to Lederle and Co. for the supply of free "triamcinolone," and to Armour and Co. for the generous supply of A.C.T.H. gel.

REFERENCES

Appleby, J. I., Gibson, G., Norymberski, J. K., and Stubbs, R. D. (1955). Biochem. J., 60, 453.

Bernstein, S., Lenhard, R. H., Allen, W. S., Heller, M., Littell, R., Stolar, S. M., Feldman, L. I., and Blank, R. H. (1956). J. Amer. chem. Soc., 78, 5693.

Cope, C. L. (1956). Brit. med. J., 2, 193.

DiRaimondo, V. C., and Forsham, P. H. (1958). Metabolism, 7, 5. Jailer, J. W., Gold, J. J., and Wallace, E. Z. (1954). Amer. J. Med., 16,340 .

Liddle, G. W., Richard, J. E., and Peterson, R. E. (1955). Endocrinology, 57,594

M.R.C. Committee on Clinical Endocrinology (1951). Lancet, 2, 585. Norymberski, J. K., Stubbs, R. D., and West, H. F. (1953). Ibid., $1,1276$.

Peterson, R. E., Karrer, A., and Guerra, S. L. (1957). Analyt. Chem., 29, 144.

Sayers, G., and Sayers, M. A. (1948). Recent Progr. Hormone Res.,

Silber, R. H., and Porter, C. C. (1954). J. biol. Chem., $210,923$. 